\title{
Design of fully automatic antibiotics evaluation machine by
}

\section{cylinder-plate method/turbidity method}

\author{
Lijian Zhang ${ }^{1, a}$, Jinlan Zhang ${ }^{1, b}$, Tingting Wang ${ }^{2}$ \\ ${ }^{1}$ College of Electrical Engineering, Binzhou Polytechnic,Shandong,256600,China \\ ${ }^{2}$ Bohai Piston Co., Ltd, Binzhou,Shandong,256600,China \\ axianruibrother@163.com, b41350992@qq.com, c1653226361@qq.com
}

Keywords: Sample meter; Constant temperature; Automatic on-line inspection

\begin{abstract}
: the utility model relates to microorganisms antibiotic titer test environment, automatic sampling, in the process of cultivation and automatic online detection field, especially relates to antibiotics tube plate method and turbidity method fully automatic meter, for experiments in purification, constant temperature, medium automatic constant temperature, automatic test, automatic steel tube and in view of the high temperature more uniform temperature in the process of cultivation in vitro bacteria liquid turbidity to implement on-line detection of automation equipment.
\end{abstract}

\section{Introduction}

At present, the antibiotic tube plate method and turbidity method verification are mostly need in a separate sterile, constant temperature laboratory environment, need a variety of instruments and equipment as well as the cumbersome manual operation, so increased the error probability of the experimental results, affected the accuracy of determination results; In addition, the tube temperature error is large, and bacterial growth had close relationship with the environment temperature, so the test tube temperature heterogeneous type directly affects the bacterial growth rate, thus affect the turbidity result; Moreover, the purification of the microbiological tests need to be independent, constant temperature environment, set up independent sterile purification laboratories, not only need to increase the test space, and add the test cost.

This equipment provides a kind of automatic constant temperature test space purification system of automatic constant temperature, automatic tube plate method, medium plate (include: media, automatic steel tube, automatic liquid), sample turbidity method of automatic, automatic stirring, automatic constant temperature medium, on-line automatic continuous measurement function of antibiotic tube plate method and turbidity method fully automatic measuring instrument. It combines many functions in one instrument, realized the full automation operation, remove the cumbersome manual operation, to eliminate the error factors, improves the accuracy and reliability of the experimental results.

\section{Equipment}

Antibiotics all-in-one evaluation by cylinder-plate method/turbidity method fully automatic, including lab automatic constant temperature purification plant, steel pipe automatic placement device, automatic sampling device, automatic constant temperature water bath device, AGAR 
culture medium bracket device, automatic turbidity measurement device, eight part motion platform device and control system.

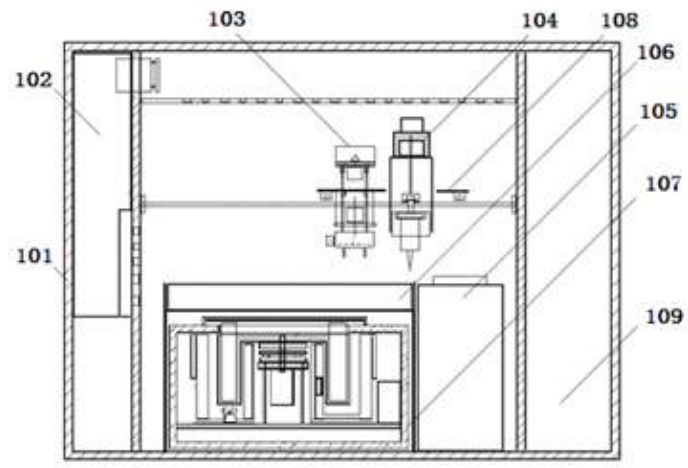

Fig.1 schematic

Lab automatic constant temperature, purification plant is located in the experimental chamber, steel pipe automatic device, automatic sampling device is placed in the experiment in motion platform, medium automatic constant temperature water bath device in the lab at, AGAR bracket device is located in the experiment in turbidity automatic measuring devices, motion platform device is located in the central lab, control system installed in a cabin on the right side of experiment.

\section{Lab automatic constant temperature}

Lab automatic constant temperature, purification device is mainly composed of air purification filter, blower, constant temperature heating device, uniform air distribution plate, temperature compensation device, return air device; Fan installed in the outside of the return air device, for the following air purification filters, heating device installed in air purification filters out FengKouChu, even with the top of the air distribution plate installed in the lab, return air device is located in the lab on the wall.

\section{Steel tube placement device automatically}

Steel pipe automatic placement device is mainly composed of steel pipe storage cup, steel tube device, steel pipe, steel pipe distributor guides including the upper, middle, lower, middle of shifter lever, electromagnet, lifting gear, steel pipe store at the bottom of the cup set with four or six steel pipe guide, its bottom end respectively embedded in the steel pipe distributor.

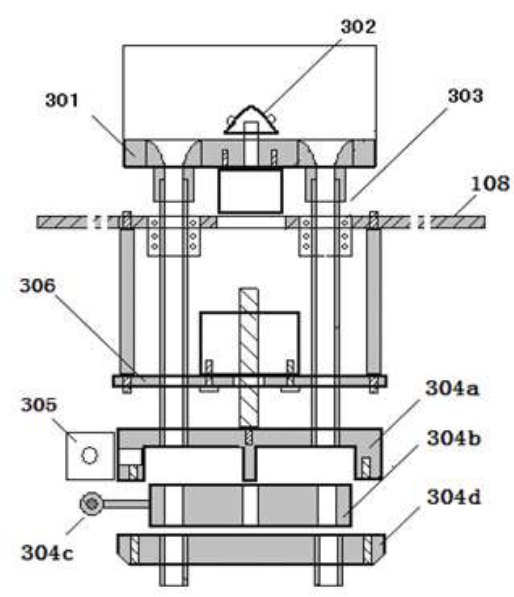

Fig.2 steel pipe automatic placement device schematic diagram 


\section{Automatic device}

Automatic washer add sample device is mainly composed of washer lifting system and the system composition.

Add pipette lifting system is mainly composed of lifting system fixed plate washer, linear motion guide, lifting gear washer, connection of linear motion guide device is fixed in the plate on the back of the elevator system, add pipette lifting gear fixed on the fixed plate lifting system, lifting system fixed on the motion platform, and pipette connection plate washer fixed on the lifting device.

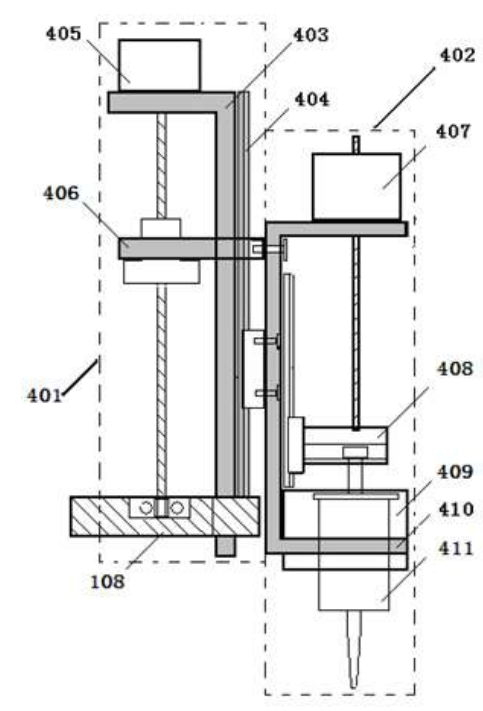

Fig.3 Schematic diagram of automatic sample adding device

The sample injector system mainly by shifting latch lifting device of liquid, liquid device plug fixing device shift, moving liquid device fixing frame, sample retainer plate, shift for liquid composition, liquid and latch lifting device fixed on the sample retainer plate, moving liquid device fixing frame stuck at the lower end of the sample retainer plate, the pipettes are embedded in the moving liquid device fixing frame and the bolt embedded in pipetting bolt fixing device and injector fixing plate fixed on the sample adding device on the connecting plate.

\section{Medium automatic thermostatic water bath device}

Culture based automatic constant temperature water bath device is mainly composed of a water bath, water bath bracket, an extension plate, a guide rail, a heating plate, a heat-insulating layer, a temperature sensor sleeve, the heating plate fixed at the bottom of the bath, temperature sensor sleeve is fixed inside the water bath, in bath and prolong plate are respectively provided with training base cup hole and a sample cup hole, water bath of bottom and around the bread with insulation layer, bath is connected through a bracket and a guide rail is fixed on the bottom plate of the whole instrument, training based automatic constant temperature water bath device as a whole can move forward and backward along the guideway.

\section{Flat tray bracket}

Petri dish bracket device mainly by the plate bracket, slides, a front baffle, a rear baffle plate, a horizontal bead, a bracket supporting plate; bracket plate on both sides of the fixed a pallet slide, front and rear ends have front baffle plate and the rear baffle plate, left and right two plate slideway are respectively fixed on the left and the right upper part of the bracket of the support plate, bracket of the support plate and the lower end of the instrument structure bottom plate is connected, 
horizontal bead in in the middle of the plate bracket, flat plate, the bracket of the device as a whole can move back and forth along the slideway.

\section{Turbidity automatic measuring device}

The turbidity measurement device is composed of the main turbidity detection box, the absorption cell rotating device, the automatic constant temperature circulating tank, the stirring device and the photoelectric detection device.

\section{Absorption cell rotating device}

Absorption pool rotation device is mainly by absorption cell rotating disk, absorption pool rotation groove, a motor, absorption pool rotary table bracket, a rotating shaft, absorption pool rotating disc bracket and the motor are respectively fixed on the automatic detection of the soleplate of the box body, motor directly or indirectly with the rotary shaft is connected, thrust bearing in absorption pool a rotary disk and absorption cell rotating disc bracket, 40 absorption cell are respectively inserted in the absorption pool rotation groove.

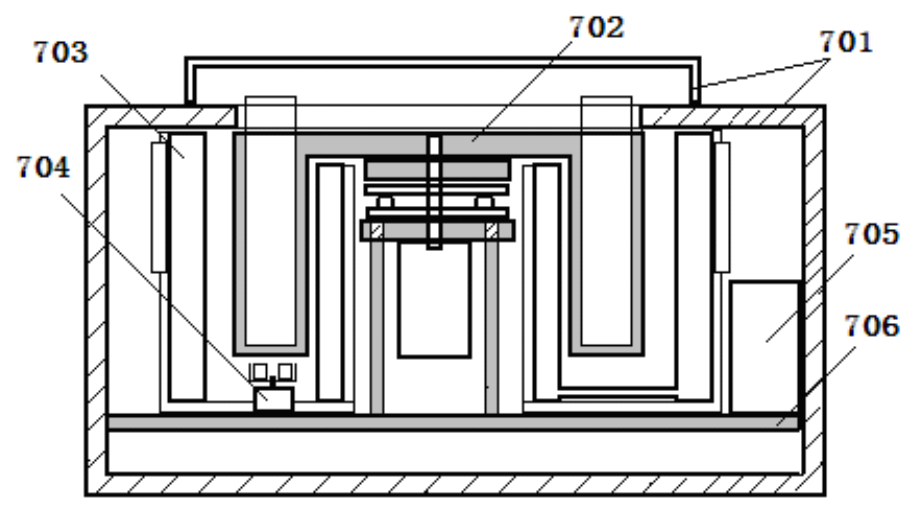

Fig.4 absorption cell rotating device

\section{Double loop automatic constant temperature device}

Double loop automatic constant temperature device positioned in the absorption both inner and outer sides of the pool rotation slot rotation trajectory, mainly by liner, bile, circulation device, temperature control device, a guide tube, a heat preservation layer, between the liner and the outer liner formation thermostatic clamping groove, one end of which is connected through a catheter, the other end connected with circulation device, temperature control device is arranged at the outer side of the outer liner, insulation layer is fixed in the tank side and the outer container outer side surface and the bottom surface. 


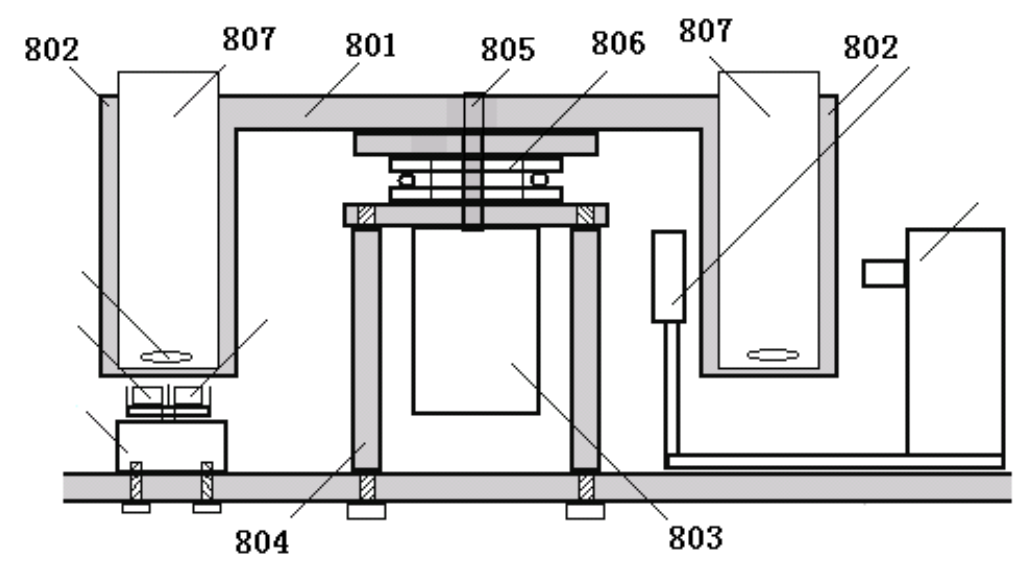

Fig.5 double loop automatic constant temperature device

\section{Automatic mixing device}

The device is composed of the motor, the magnet piece and the rotor. The motor is mounted in the slot of the motor shaft and the rotor is placed in the absorption cell.

\section{Photoelectric detection device}

Photoelectric detection device positioned in the absorption on the inner and outer sides of the groove of the rotation of the pool below the rotation trajectory, is mainly composed of a light source, a photoelectric detector, a light source is composed of light emitting body, a reflector, a monochromatic includes a prism or grating diffraction, a filter, a collimating lens, wherein the luminous body can be as full wavelength long life lamps or single wave long life light source, the use of wavelength adjustable.

\section{Motion platform}

On the lead rail of the two connected through the outer side of the platform motion device is mainly composed of the $\mathrm{X}$ axis guide rail, a $\mathrm{Y}$ axis guide rail, a moving plate, $\mathrm{X}$ shaft and $\mathrm{Y}$ shaft of the motor, a front slide plate, the slide plate, two $X$ axis guide rail which is parallel to the lead screw is fixed on the experimental class of left and right wall, the $\mathrm{X}$ axis motor is fixed on the bulkhead screw or synchronous belt and the rear sliding plate, the slide plate and the slide plate are respectively placed on the $\mathrm{X}$ axis of a front and a back guide rails, two $\mathrm{Y}$ axis is parallel to the guide way fixed before the slide plate and the slide plate, the plate to move through a bearing fixed on the $\mathrm{Y}$ axis, $\mathrm{Y}$ axis motor fixed sliding plate, through the synchronous belt and the front skateboard, mobile plate connection.

\section{Control system}

The control system is composed of motion control system, electrical control system, temperature control system, photoelectric detection and control system, touch screen and power device..

Motion control system mainly comprises a disk stepper motor, a total of 45 station accurate positioning movement; freely telescopic motion of the arm, sample front extending to the sample position, the sample after the completion of the return origin; peristaltic pump and movement, starting from 0 station, according to the bacterium on touch screen set in advance of the determination method, according to certain rules in order to complete glass dishes of the sample. These three stepper motor in the machine when the electric, automatic return to the origin of the operation. 
Temperature control system, the use of the thermocouple to take the experimental cabin temperature, PID algorithm for heating belt control, the experimental warehouse control in the setting temperature.

The touch screen uses the Beijing MCGS product, completes the data storage operation, uses the script, completes many kinds of calibration method programming work.

\section{Conclusion}

This instrument integrates many functions, realizes the operation automation, relieves the complicated manual operation, eliminates many error factors and improves the accuracy and reliability of the experimental results.. The equipment has the following advantages for the existing technology:

1) realize the automatic constant temperature purification air supply function, and save the test space at the same time;

2) dual cycle type thermostatic temperature turbidity culture device, temperature uniform, increase the comparability of test tube;

3) a high degree of automation, the realization of the test of the same, standardization, a decrease of many error factors, improves the accuracy and reliability of the experimental results, and at the same time, save manpower, material resources and test time;

4) the function of the whole and one machine can be used for the test of the two methods of antibiotic tube plate and turbidity.

\section{Acknowledgement}

This research was financially supported by Colleges and universities in shandong province department of education scientific research and development projects.

Project name: intravenous infusion of the control system of automatic dispensing apparatus development with item number: J14LB57.

\section{Reference}

[1] Shi Yuhui, Yang Yongyi, Ma Aiping. The maintenance and indoor quality control of the automatic sample [J]. China transfusion journal, 2005, 18 (5): 406

Wang Chao. Study on [J]. [2] automation instrument.2013 a new type of intelligent dispensing equipment (7)

[3] Li Xin welcome, Li Xihe, Wang Jing et al. Development of biochemical analyzer [J]. medical equipment, 2012 tenth: $6 \sim 7$

[4] Wang Shimin. Design of automatic sample [J]. equipment for micro - powder.2013,26 (5): 123-126

[5] Huang Caixia, Qi Jingjing, Huang Libin. Design [J]. computer measurement and control.2013 (21) for petrochemical pharmaceutical automatic preparation system based on PLC 